\title{
On Hesitant Fuzzy Reducible Weighted Bonferroni Mean and Its Generalized Form for Multicriteria Aggregation
}

\author{
Wei Zhou \\ International Business School, Yunnan University of Finance and Economics, Kunming 650221, China \\ Correspondence should be addressed to Wei Zhou; zw453@163.com
}

Received 21 October 2013; Accepted 14 February 2014; Published 25 March 2014

Academic Editor: Ching-Jong Liao

Copyright ( 2014 Wei Zhou. This is an open access article distributed under the Creative Commons Attribution License, which permits unrestricted use, distribution, and reproduction in any medium, provided the original work is properly cited.

\begin{abstract}
Due to convenience and powerfulness in dealing with vagueness and uncertainty of real situation, hesitant fuzzy set has received more and more attention and has been a hot research topic recently. To differently process and effectively aggregate hesitant fuzzy information and capture their interrelationship, in this paper, we propose the hesitant fuzzy reducible weighted Bonferroni mean (HFRWBM) and present its four prominent characteristics, namely, reductibility, monotonicity, boundedness, and idempotency. Then, we further investigate its generalized form, that is, the generalized hesitant fuzzy reducible weighted Bonferroni mean (GHFRWBM). Based on the discussion of model parameters, some special cases of the HFRWBM and GHFRWBM are studied in detail. In addition, to deal with the situation that multicriteria have connections in hesitant fuzzy information aggregation, a threestep aggregation approach has been proposed on the basis of the HFRWBM and GHFRWBM. In the end, we apply the proposed aggregation operators to multicriteria aggregation and give an example to illustrate our results.
\end{abstract}

\section{Introduction}

In order to better understand the vagueness and uncertainty of the real world and thus be able to explain it, the fuzzy set (FS) theory has been extended and generalized to many other forms, such as interval-valued fuzzy set [1], type-2 fuzzy set [2,3], fuzzy multiset [4], intuitionistic fuzzy set [5], interval-valued intuitionistic fuzzy set [6], and hesitant fuzzy set [7]. Due to convenience and powerfulness in dealing with vagueness and uncertainty of real situation, hesitant fuzzy set has received more and more attention from researchers and obtained some significant research results [8-12]. To aggregate the hesitant fuzzy information, a lot of aggregation operators have been developed and investigated, such as the hesitant fuzzy averaging aggregation (HFA) operator, the hesitant fuzzy geometric aggregation (HFGA) operator, the hesitant fuzzy ordered weighted averaging (HFOWA) operator, the hesitant fuzzy ordered weighted geometric (HFOWG) operator, and the hesitant fuzzy hybrid aggregation (HFHA) operator [13].

The Bonferroni mean (BM) was introduced by Bonferroni [14] six decades ago but has been a hot research topic recently for its important role in the information aggregation, which can provide for the aggregation lying between the max and min operators and logical "oring" and "anding" operators. A prominent characteristic of BM is that it not only considers the importance of each criterion but also reflects the interrelationship of the individual criterion [15]. The extended BMs include the generalized Bonferroni mean [16], the intuitionistic fuzzy Bonferroni mean [17], the intervalvalued intuitionistic fuzzy Bonferroni mean [18], the revised Bonferroni mean [19], the intuitionistic fuzzy geometric Bonferroni mean [12], the hesitant fuzzy geometric Bonferroni mean, and the hesitant fuzzy Choquet geometric Bonferroni mean [20].

In reality, more differences are required to be considered in the multicriteria aggregation process due to different functions and influences among arguments in practical applications, especially, in hesitant fuzzy environment. To overcome this limitation, some weighted BMs have been developed, such as the intuitionistic fuzzy weighted Bonferroni mean [17], the interval-valued intuitionistic fuzzy weighted Bonferroni mean [18], the intuitionistic fuzzy normalized weighted Bonferroni mean [12], and the revised generalized weighted Bonferroni mean [19]. To suit the hesitant fuzzy environment, Zhou and $\mathrm{He}$ [12] proposed the weighted hesitant fuzzy 
Bonferroni mean, and Zhu et al. [20] defined the weighted hesitant fuzzy geometric Bonferroni mean. However, a problem arises; that is, the HFBM, HFGBM, and HFCGBM cannot be obtained, respectively, when all the weights of the aggregated arguments are the same, which means the above weighted hesitant fuzzy BMs have not the reducibility that is a basic property among the weighted operators.

With the analysis above, in this paper, we propose the hesitant fuzzy reducible weighted Bonferroni mean and its generalized form and organize this paper as follows. Some basic concepts and operations are reviewed in Section 2. Sections 3 and 4, respectively, propose the hesitant fuzzy reducible weighted Bonferroni mean (HFRWBM) and the generalized hesitant fuzzy reducible weighted Bonferroni mean (GHFRWBM) and study their desirable properties. In Section 5, based on HFRWBM and GHFRWBM, a threestep aggregation approach has been proposed to deal with the hesitant fuzzy multicriteria aggregation, and a practical example is provided to demonstrate their application. The paper ends in Section 6 with concluding remarks.

\section{Preliminaries}

The Bonferroni mean (BM) and the generalized Bonferroni mean (GBM) were originally introduced by Bonferroni [14] and Zhou and $\mathrm{He}$ [16]. As two extensions of the arithmetic average, they are two very useful multicriteria aggregation operators, which consider the interrelationships among arguments. Now we introduce the concepts of the BM and GBM as follows.

Definition 1 (see [14]). Let $p, q \geq 0$, and $a_{i}(i=1,2, \ldots, n)$ be a collection of nonnegative numbers. Then, the Bonferroni mean $(B M)$ is defined as

$$
\operatorname{BM}^{p, q}\left(a_{1}, a_{2}, \ldots, a_{n}\right)=\left(\frac{1}{n(n-1)} \sum_{\substack{i, j=1 \\ i \neq j}}^{n} a_{i}^{p} a_{j}^{q}\right)^{1 /(p+q)} .
$$

Definition 2 (see [16]). Let $p, q, r \geq 0$, and $a_{i}(i=1,2, \ldots, n)$ be a collection of nonnegative numbers. Then, the generalized Bonferroni mean (GBM) is defined as

$$
\begin{aligned}
\mathrm{GBM}^{p, q, r} & \left(a_{1}, a_{2}, \ldots, a_{n}\right) \\
= & \left(\frac{1}{n(n-1)(n-2)} \sum_{\substack{i, j, k=1 \\
i \neq j \neq k}}^{n} a_{i}^{p} a_{j}^{q} a_{k}^{r}\right)^{1 /(p+q+r)} .
\end{aligned}
$$

It is obvious that the GBM could reduce to the BM if $r=0$, and the GBM can represent the interrelationship of any three criteria. However, it is noted that the above BM and GBM can only deal with the situation that the arguments are represented by real number and ignore the weight vector of the aggregated arguments. To deal with this issue, $\mathrm{Xu}$ and Yager [17] extended BM to the fuzzy environment and proposed the following intuitionistic fuzzy Bonferroni mean
(IFBM) and intuitionistic fuzzy weighted Bonferroni mean (IFWBM).

Definition 3 (see [17]). Let $p, q, r \geq 0$, and $\alpha_{i}(i=1,2, \ldots, n)$ be a collection of intuitionistic fuzzy values. The intuitionistic fuzzy Bonferroni mean (IFBM) and the intuitionistic fuzzy weighted Bonferroni mean (IFWBM) are, respectively, defined as

$$
\begin{aligned}
& \operatorname{IFBM}^{p, q}\left(\alpha_{1}, \alpha_{2}, \ldots, \alpha_{n}\right) \\
& \quad=\left(\frac{1}{n(n-1)} \bigoplus_{i, j=1, i \neq j}^{n}\left(\alpha_{i}^{p} \otimes \alpha_{j}^{q}\right)\right)^{1 /(p+q)}, \\
& \quad=\left(\frac{1}{n(n-1)} \bigoplus_{i, j=1, i \neq j}^{n}\left(\left(w_{i} \alpha_{i}^{p}\right) \otimes\left(w_{j} \alpha_{j}^{q}\right)\right)\right)^{1 /(p+q)} .
\end{aligned}
$$

Recently, Torra and Narukawa [7] originally proposed the hesitant fuzzy set which is a more general fuzzy set and permits the membership having a set of possible values.

Definition 4 (see [13]). Let $X$ be a fixed set; a hesitant fuzzy set (HFS) on $X$ is in terms of a function that when applied to $X$ returns a subset of $[0,1]$.

To be easily understood, Xia and $\mathrm{Xu}$ [13] expressed the HFS by a mathematical symbol:

$$
E=\left\{\left\langle x, h_{E}(x)\right\rangle \mid x \in X\right\}
$$

where $h_{E}(x)$ is a set of some values in $[0,1]$ and called the hesitant fuzzy element (HFE), denoting the possible membership degrees of the element $x$ to the set $E$.

Definition 5 (see [13]). For a $\operatorname{HFE} h, s(h)=\sum_{\gamma \in h} \gamma / l(h)$ is called the score function of $h$, where $l(h)$ is the number of values in $h$ and $\gamma$ is element of the hesitant fuzzy set $h$. For two HFEs, $h_{1}$ and $h_{2}$, if $s\left(h_{1}\right)>s\left(h_{2}\right)$, then $h_{1}>h_{2}$; if $s\left(h_{1}\right)=s\left(h_{2}\right)$, then $h_{1}=h_{2}$.

There are some operational laws about any three HFEs; $h$, $h_{1}$, and $h_{2}$ are as below:

(1) $h^{\lambda}=\bigcup_{\gamma \in h}\left\{\gamma^{\lambda}\right\}, \lambda>0$,

(2) $\lambda h=\bigcup_{\gamma \in h}\left\{1-(1-\gamma)^{\lambda}\right\}, \lambda>0$,

(3) $h_{1} \otimes h_{2}=\bigcup_{\gamma_{1} \in h_{1}, \gamma_{2} \in h_{2}}\left\{\gamma_{1} \gamma_{2}\right\}$

(4) $h_{1} \oplus h_{2}=\bigcup_{\gamma_{1} \in h_{1}, \gamma_{2} \in h_{2}}\left\{\gamma_{1}+\gamma_{2}-\gamma_{1} \gamma_{2}\right\}$

(5) $\lambda\left(h_{1} \oplus h_{2}\right)=\lambda h_{1} \oplus \lambda h_{2}$,

(6) $\left(h_{1} \otimes h_{2}\right)^{\lambda}=h_{1}^{\lambda} \otimes h_{2}^{\lambda}$. 
By referencing to the IFBM and IFWBM, the following hesitant fuzzy Bonferroni mean (HFBM) and the hesitant fuzzy weighted Bonferroni mean (HFWBM) could be defined:

$$
\begin{aligned}
& \operatorname{HFBM}^{p, q}\left(h_{1}, h_{2}, \ldots, h_{n}\right) \\
& \quad=\left(\frac{1}{n(n-1)} \bigoplus_{i, j=1, i \neq j}^{n}\left(h_{i}^{p} \otimes h_{j}^{q}\right)\right)^{1 /(p+q)}, \\
& \operatorname{HFWBM}^{p, q}\left(h_{1}, h_{2}, \ldots, h_{n}\right) \\
& =\left(\frac{1}{n(n-1)} \bigoplus_{i, j=1, i \neq j}^{n}\left(\left(w_{i} h_{i}^{p}\right) \otimes\left(w_{j} h_{j}^{q}\right)\right)\right)^{1 /(p+q)} .
\end{aligned}
$$

According to the classic arithmetic averaging (AA) and weighted averaging (WA) operators, the intuitionistic fuzzy arithmetic averaging (IFAA) and intuitionistic fuzzy weighted averaging (IFWA) operators, and the hesitant fuzzy arithmetic averaging (HFAA) and hesitant fuzzy weighted averaging (HFWA), it is found that the following reducibility is the basic property among these operators; that is, if $w_{i}=$ $1 / n, i=1,2, \ldots, n$, then AA = WA, IFAA = IFWA, and HFAA $=$ HFWA.

It is obvious that the above HFBM and HFWBM are not equal when $w_{i}=1 / n$ and have not the reducibility. To deal with this issue, Xia et al. [19] proposed the generalized weighted Bonferroni mean (GWBM) and the corresponding generalized hesitant fuzzy weighted Bonferroni mean (GHFWBM) based on the GWBM, which are described as follows:

$$
\begin{aligned}
& \operatorname{GWBM}^{p, q, r}\left(a_{1}, a_{2}, \ldots, a_{n}\right) \\
& =\left(\sum_{i, j, k=1}^{n} w_{i} w_{j} w_{k} a_{i}^{p} a_{j}^{q} a_{k}^{r}\right)^{1 /(p+q+r)}, \\
& \operatorname{GHFWBM}^{p, q, r}\left(h_{1}, h_{2}, \ldots, h_{n}\right) \\
& =\left(\bigoplus_{i, j, k=1,}^{n} w_{i} w_{j} w_{k} h_{i}^{p} h_{j}^{q} h_{k}^{r}\right)^{1 /(p+q+r)} .
\end{aligned}
$$

However, another question arises; that is, the above GWBM and GHFWBM just consider the whole correlationship between the criterion and all criteria and cannot reflect the interrelationship between the individual criterion and other criteria which is the main advantage of the BM [15]. To overcome this drawback and introduce the reducibility, we propose the hesitant fuzzy reducible weighted Bonferroni mean (HFRWBM) and its generalized form called generalized hesitant fuzzy reducible weighted Bonferroni mean (GHFRWBM) in next sections.

\section{Hesitant Fuzzy Reducible Weighted Bonferroni Mean (HFRWBM)}

In multicriteria aggregation, the performance of an alternative under a criterion may be presented by several possible values. To aggregate all the possible values of an alternative under the criteria and reflect the interrelationship between the individual criterion and other criteria, we give a new hesitant fuzzy weighted Bonferroni mean with the reducibility, which is defined as follows.

Definition 6. Let $p, q \geq 0$, and $h_{i}(i=1,2, \ldots, n)$ be a collection of HFEs with the weight vector $w=\left(w_{1}, w_{2}, \ldots, w_{n}\right)$ such that $w_{i} \geq 0, w_{i} \neq 1$, and $\sum_{i=1}^{n} w_{i}=1$. If

$$
\begin{aligned}
\operatorname{HFRWBM}_{w}^{p, q}\left(h_{1}, h_{2}, \ldots, h_{n}\right) \\
=\left(\bigoplus_{i, j=1, i \neq j}^{n} \frac{w_{i} w_{j}}{1-w_{i}}\left(h_{i}^{p} \otimes h_{j}^{q}\right)\right)^{1 /(p+q)},
\end{aligned}
$$

then $\operatorname{HFRWBM}^{p, q}$ is called a hesitant fuzzy reducible weighted Bonferroni mean (HFRWBM).

Then, we can transform the HFRWBM in to the interrelationship HFRWBM form as follows:

$$
\begin{aligned}
& \operatorname{HFRWBM}_{w}^{p, q}\left(h_{1}, h_{2}, \ldots, h_{n}\right) \\
& =\left(\bigoplus_{i=1}^{n} w_{i} h_{i}^{p}\left(\bigoplus_{j=1, i \neq j}^{n} \frac{w_{j}}{1-w_{i}} h_{j}^{q}\right)\right)^{1 /(p+q)} .
\end{aligned}
$$

We see that the term $\oplus_{j=1, i \neq j}^{n}\left(w_{j} /\left(1-w_{i}\right)\right) h_{j}^{q}$ is the weighted power average satisfaction of all criteria except $H_{i}$, and $\oplus_{j=1, i \neq j}^{n}\left(w_{j} /\left(1-w_{i}\right)\right)=1$. We denote the term as $v_{i}^{q}$. Thus

$$
\begin{aligned}
\operatorname{HFRWBM}_{w}^{p, q}\left(h_{1}, h_{2}, \ldots, h_{n}\right) \\
=\left(\bigoplus_{i=1}^{n} w_{i}\left(h_{i}^{p} \otimes v_{i}^{q}\right)\right)^{1 /(p+q)} .
\end{aligned}
$$

Here then $v_{i}^{q}$ is the weighted power average satisfaction to all criteria except $H_{i}$, and $\operatorname{HFRWBM}^{p, q}$ represents the weighted interrelationship between the individual criterion $h_{i}$ and other criteria $v_{i}$ which is similar to the BM.

Based on the operational law of HFRWBM, we further derive the following results.

Theorem 7. Let $p, q \geq 0$, and $h_{i}(i=1,2, \ldots, n)$ be a collection of HFEs with the weight vector $w=\left(w_{1}, w_{2}, \ldots, w_{n}\right)$ such that $w_{i} \geq 0, w_{i} \neq 1$ and $\sum_{i=1}^{n} w_{i}=1$; then the aggregation value by using the HFRWBM is a HFE, and

$$
\begin{aligned}
& \operatorname{HFRWBM}_{w}^{p, q}\left(h_{1}, h_{2}, \ldots, h_{n}\right) \\
& =\left(\bigoplus_{i, j=1, i \neq j}^{n} \kappa_{i j}\right)^{1 /(p+q)} \\
& =\bigcup_{k_{i, j} \in \kappa_{i j, i \neq j}}\left\{\left(1-\prod_{i, j=1, i \neq j}^{n}\left(1-k_{i, j}\right)\right)^{1 /(p+q)}\right\} \text {, }
\end{aligned}
$$

where $\kappa_{i j}=\left(w_{i} w_{j} /\left(1-w_{i}\right)\right)\left(h_{i}^{p} \otimes h_{j}^{q}\right)$ can be considered as "bonding satisfaction" factor used as a calculation unit, capturing the connection between $h_{i}$ and $h_{j}$. 
Proof. By the operational laws (1)-(6) of HFEs, we obtain

$$
\begin{aligned}
\kappa_{i j}= & \frac{w_{i} w_{j}}{1-w_{i}}\left(h_{i}^{p} \otimes h_{j}^{q}\right) \\
= & \frac{w_{i} w_{j}}{1-w_{i}} \bigcup_{\substack{r_{i} \in h_{i} \\
r_{j} \in h_{j}}}\left(r_{i}^{p} r_{j}^{q}\right) \\
= & \bigcup_{\substack{r_{i} \in h_{i} \\
r_{j} \in h_{j}}}\left(1-\left(1-r_{i}^{p} r_{j}^{q}\right)^{w_{i} w_{j} /\left(1-w_{i}\right)}\right),
\end{aligned}
$$

which is also a HFE; then (8) can be written as

$$
\operatorname{HFRWBM}_{w}^{p, q}\left(h_{1}, h_{2}, \ldots, h_{n}\right)=\left(\bigoplus_{i, j=1, i \neq j}^{n} \kappa_{i j}\right)^{1 /(p+q)}
$$

Furthermore, from the operational laws of HFEs, we have

$$
\left(\bigoplus_{i, j=1, i \neq j}^{n} \kappa_{i j}\right)=\bigcup_{k_{i, j} \in \kappa_{i j, i \neq j}}\left\{1-\prod_{i, j=1, i \neq j}^{n}\left(1-k_{i, j}\right)\right\} .
$$

Therefore,

$$
\begin{aligned}
\operatorname{HFRWBM}_{w}^{p, q}\left(h_{1}, h_{2}, \ldots, h_{n}\right) \\
=\left(\bigoplus_{i, j=1, i \neq j}^{n} \kappa_{i j}\right)^{1 /(p+q)} \\
=\bigcup_{k_{i, j} \in \kappa_{i, i \neq j}}\left\{\left(1-\prod_{i, j=1, i \neq j}^{n}\left(1-k_{i, j}\right)\right)^{1 /(p+q)}\right\} .
\end{aligned}
$$

Then the proof is completed.

From (8), we can see that the HFRWBM can fully represent the interrelationship between the individual criterion and other criteria by two types of conjunction calculations, that is, $\oplus$ and $\otimes$. Moreover, after the original data being operated by the conjunction calculations, their values have been changed. In multicriteria aggregation problems, the HFRWBM can result in the advantage of the BM considering the individual criterion and other criteria in aggregation process, which can take much more hesitant fuzzy information into account.

The $\kappa_{i j}$ can be considered as "bonding satisfaction" factor, which is the basic calculation unit of the HFRWBM. According to (8), we can derive the reducibility of HFRWBM and other useful properties as follows.

Property 1 (reducibility). Let $p, q \geq 0$, and $h_{i}(i=1,2, \ldots, n)$ be a collection of HFEs with the weight vector $w=$ $(1 / n, 1 / n, \ldots, 1 / n)$; then

$$
\operatorname{HFRWBM}_{w}^{p, q}\left(h_{1}, h_{2}, \ldots, h_{n}\right)=\operatorname{HFBM}^{p, q}\left(h_{1}, h_{2}, \ldots, h_{n}\right) .
$$

Proof. Since $w_{i}=1 / n(i=1,2, \ldots, n)$, then, by Definition 6, we have

$$
\begin{aligned}
\operatorname{HFRWBM}_{w}^{p, q}\left(h_{1}, h_{2}, \ldots, h_{n}\right) \\
=\left(\frac{1}{n(n-1)} \bigoplus_{i, j=1, i \neq j}^{n}\left(h_{i}^{p} \otimes h_{j}^{q}\right)\right)^{1 /(p+q)} \\
=\operatorname{HFBM}^{p, q}\left(h_{1}, h_{2}, \ldots, h_{n}\right)
\end{aligned}
$$

which complete the proof of the property.

Property 2 (monotonicity). Let $h_{\alpha}=\left\{h_{\alpha 1}, h_{\alpha 2}, \ldots, h_{\alpha n}\right\}$ and $h_{\beta}=\left\{h_{\beta 1}, h_{\beta 2}, \ldots, h_{\beta n}\right\}$ be two collections of HFEs, if, for any $\gamma_{\alpha i} \in h_{\alpha i}$ and $\gamma_{\beta i} \in h_{\beta i}$, we have $\gamma_{\alpha i} \leq \gamma_{\beta i}$ for all $i(i=1,2, \ldots, n)$, and thus

$$
\begin{aligned}
& \operatorname{HFRWBM}_{w}^{p, q}\left(h_{\alpha 1}, h_{\alpha 2}, \ldots, h_{\alpha n}\right) \\
& \leq \operatorname{HFRWBM}_{w}^{p, q}\left(h_{\beta 1}, h_{\beta 2}, \ldots, h_{\beta n}\right) .
\end{aligned}
$$

Proof. Using the terms above and (11), we have

$$
\begin{aligned}
(1- & \left.\prod_{i, j=1, i \neq j}^{n}\left(1-k_{\alpha_{i, j}}\right)\right)^{1 /(p+q)} \\
& \leq\left(1-\prod_{i, j=1, i \neq j}^{n}\left(1-k_{\beta_{i, j}}\right)\right)^{1 /(p+q)} .
\end{aligned}
$$

Then,

$$
\begin{aligned}
& \left(\bigoplus_{i, j=1, i \neq j}^{n} \kappa_{\alpha_{i j}}\right)^{1 /(p+q)} \\
& =\bigcup_{\alpha_{\alpha_{i j}} \in \kappa_{\alpha_{i j, i \neq j}}}\left\{\left(1-\prod_{i, j=1, i \neq j}^{n}\left(1-k_{\alpha_{i j}}\right)\right)^{1 /(p+q)}\right\} \\
& \leq\left(\bigoplus_{i, j=1, i \neq j}^{n} \kappa_{\beta_{i j}}\right)^{1 /(p+q)} \\
& =\bigcup_{k_{\beta_{i j}} \in \kappa_{\beta_{i j, i \neq j}}}^{n}\left\{\left(1-\prod_{i, j=1, i \neq j}^{n}\left(1-k_{\beta_{i j}}\right)\right)^{1 /(p+q)}\right\},
\end{aligned}
$$

which complete the proof of the property.

Property 3 (boundedness). Let $p, q \geq 0$, and $h_{i}(i=$ $1,2, \ldots, n)$ be a collection of HFEs with the weight vector $w=\left(w_{1}, w_{2}, \ldots, w_{n}\right)$ such that $w_{i} \geq 0, w_{i} \neq 1$, and $\sum_{i=1}^{n} w_{i}=1$; we have

$$
h^{-} \leq \operatorname{HFRWBM}_{w}^{p, q}\left(h_{1}, h_{2}, \ldots, h_{n}\right) \leq h^{+},
$$

where $h^{-}$and $h^{+}$are lower and upper bounds, respectively [8], with $h^{-}=\min _{i}\left\{\gamma_{i} \mid \gamma_{i} \in h_{i}\right\}$ and $h^{+}=\max _{i}\left\{\gamma_{i} \mid \gamma_{i} \in h_{i}\right\}$. 
Proof. Since $h^{-} \leq \gamma_{i} \leq h^{+}$, for all $i$, then

$$
\begin{aligned}
\left(h^{-}\right)^{p+q}= & \left(h^{-}\right)^{p} \otimes\left(h^{-}\right)^{q} \leq h_{i}^{p} \otimes h_{j}^{q} \\
& \leq\left(h^{+}\right)^{p} \otimes\left(h^{+}\right)^{q}=\left(h^{+}\right)^{p+q}, \\
& \left(\left(h^{-}\right)^{p+q} \bigoplus_{i, j=1, i \neq j}^{n} \frac{w_{i} w_{j}}{1-w_{i}}\right)^{1 /(p+q)} \\
& \leq\left(\bigoplus_{i, j=1, i \neq j}^{n} \frac{w_{i} w_{j}}{1-w_{i}}\left(h_{i}^{p} \otimes h_{j}^{q}\right)\right)^{1 /(p+q)} \\
& \leq\left(\left(h^{+}\right)^{p+q} \bigoplus_{i, j=1, i \neq j}^{n} \frac{w_{i} w_{j}}{1-w_{i}}\right)^{1 /(p+q)} .
\end{aligned}
$$

Therefore,

$$
\begin{aligned}
h^{-} & \leq \operatorname{HFRWBM}_{w}^{p, q}\left(h_{1}, h_{2}, \ldots, h_{n}\right) \\
& =\left(\bigoplus_{i, j=1, i \neq j}^{n} \frac{w_{i} w_{j}}{1-w_{i}}\left(h_{i}^{p} \otimes h_{j}^{q}\right)\right)^{1 /(p+q)} \leq h^{+},
\end{aligned}
$$

which complete the proof of the property.

Property 4 (idempotency). Let $p, q \geq 0$, and $h_{i}(i=$ $1,2, \ldots, n)$ be a collection of HFEs with the weight vector $w=\left(w_{1}, w_{2}, \ldots, w_{n}\right)$ such that $w_{i} \geq 0, w_{i} \neq 1$, and $\sum_{i=1}^{n} w_{i}=1$. If all $h_{i}$ are equal; that is, $h_{i}=h$ for all $i$, then

$$
\operatorname{HFRWBM}_{w}^{p, q}\left(h_{1}, h_{2}, \ldots, h_{n}\right)=h .
$$

Proof. Since $h_{i}=h(i=1,2, \ldots, n)$, then

$$
\begin{aligned}
\operatorname{HFRWBM}_{w}^{p, q}\left(h_{1}, h_{2}, \ldots, h_{n}\right) \\
=\left(\bigoplus_{i, j=1, i \neq j}^{n} \frac{w_{i} w_{j}}{1-w_{i}}\left(h_{i}^{p} \otimes h_{j}^{q}\right)\right)^{1 /(p+q)} \\
=\left(\bigoplus_{i, j=1, i \neq j}^{n} \frac{w_{i} w_{j}}{1-w_{i}} h^{p+q}\right)^{1 /(p+q)} \\
=\left(h^{p+q} \bigoplus_{i, j=1, i \neq j}^{n} \frac{w_{i} w_{j}}{1-w_{i}}\right)^{1 /(p+q)}=\left(h^{p+q}\right)^{1 /(p+q)}=h,
\end{aligned}
$$

which complete the proof of the property.

Now, if we change the parameters $p$ and $q$ of the HFRWBM, then we can get some special cases as follows.

Case 1. If $p=0$ and $q=0$, based on (8), we have

$$
\operatorname{HFRWBM}_{w}^{0,0}\left(h_{1}, h_{2}, \ldots, h_{n}\right)=\bigoplus_{i=1}^{n} w_{i}=1 .
$$

Case 2. If $p=1$ and $q=0$, based on (8), we get

$$
\begin{aligned}
\operatorname{HFRWBM}_{w}^{1,0}\left(h_{1}, h_{2}, \ldots, h_{n}\right) \\
=\left(\bigoplus_{i, j=1, i \neq j}^{n} \frac{w_{i} w_{j}}{1-w_{i}}\left(h_{i}^{1} \otimes h_{j}^{0}\right)\right)^{1 / 1} \\
=\bigoplus_{i}^{n} w_{i} h_{i}=\operatorname{HFWA}\left(h_{1}, h_{2}, \ldots, h_{n}\right) .
\end{aligned}
$$

Case 3. If $p=1$ and $q=1$, then (8) is transformed as

$$
\begin{aligned}
& \operatorname{HFRWBM}_{w}^{1,1}\left(h_{1}, h_{2}, \ldots, h_{n}\right) \\
& =\left(\bigoplus_{i, j=1, i \neq j}^{n} \frac{w_{i} w_{j}}{1-w_{i}}\left(h_{i} \otimes h_{j}\right)\right)^{1 / 2},
\end{aligned}
$$

which we call a hesitant fuzzy reducible square weighted Bonferroni mean (HFRSWBM).

Case 4. If $p=2$ and $q=0$, then (8) is transformed as

$$
\operatorname{HFRWBM}_{w}^{2,0}\left(h_{1}, h_{2}, \ldots, h_{n}\right)=\left(\bigoplus_{i=1}^{n} w_{i} h_{i}^{2}\right)^{1 / 2},
$$

which we call a hesitant fuzzy Euclidean distance weighted Bonferroni mean (HFEDWBM).

Based on the above four special cases, we find that (1) Case 1 presents orthonormality of the HFRWBM operator which is an important property of the weighted aggregation operator; (2) Case 2 proves the HFWA operator is just a special case of the new hesitant fuzzy weighted aggregation operator; (3) Case 3 proposes the simplest interrelated HFRWBM operator; (4) Case 4 shows that the new HFRWBM operator could be used to compute the Euclidean distance between two HFEs. Generally, the HFRWBM ${ }^{1,1}$ is recommended to use in hesitant fuzzy multicriteria aggregation.

\section{Generalized Hesitant Fuzzy Reducible Weighted Bonferroni Mean (GHFRWBM)}

In this section, by considering the correlation of any three aggregated arguments instead of any two, we further extend the HFRWBM to a generalized form, that is, the generalized hesitant fuzzy reducible weighted Bonferroni mean (GHFRWBM), based on the HFRWBM operator. 
Definition 8. Let $p, q, r \geq 0$, and $h_{i}(i=1,2, \ldots, n)$ be a collection of HFEs with the weight vector $w=\left(w_{1}, w_{2}, \ldots, w_{n}\right)$ such that $w_{i} \geq 0, w_{i} \neq 1$, and $\sum_{i=1}^{n} w_{i}=1$. If

$$
\begin{gathered}
\operatorname{GHFRWBM}_{w}^{p, q, r}\left(h_{1}, h_{2}, \ldots, h_{n}\right) \\
=\left(\bigoplus_{i, j, t=1, i \neq j \neq t}^{n} \frac{w_{i} w_{j} w_{t}}{\left(1-w_{i}\right)\left(1-w_{i}-w_{j}\right)}\right. \\
\left.\times\left(h_{i}^{p} \otimes h_{j}^{q} \otimes h_{t}^{r}\right)\right)^{1 /(p+q+r)},
\end{gathered}
$$

then GHFRWBM ${ }^{p, q, r}$ is called a generalized hesitant fuzzy reducible weighted Bonferroni mean.

Then, we can transform the GHFRWBM in to two interrelationship forms as follows:

$$
\begin{aligned}
& \operatorname{GHFRWBM}_{w}^{p, q, r}\left(h_{1}, h_{2}, \ldots, h_{n}\right) \\
& =\left(\bigoplus_{i=1}^{n} w_{i} h_{i}^{p} \bigoplus_{j=1, i \neq j}^{n} n_{j} h_{j}^{q} \bigoplus_{t=1, t \neq i \neq j}^{n} m_{t} h_{t}^{r}\right)^{1 /(p+q+r)},
\end{aligned}
$$

$$
\begin{aligned}
& \operatorname{GHFRWBM}_{w}^{p, q, r}\left(h_{1}, h_{2}, \ldots, h_{n}\right) \\
& =\left(\bigoplus_{i=1}^{n} w_{i} h_{i}^{p}\left(\bigoplus_{j, t=1, i \neq j \neq t}^{n} u_{j, t}\left(h_{j}^{q} \otimes h_{t}^{r}\right)\right)\right)^{1 /(p+q+r)},
\end{aligned}
$$

where $n_{j}=w_{j} /\left(1-w_{i}\right), m_{t}=w_{t} /\left(1-w_{i}-w_{j}\right)$, and $u_{j, t}=$ $w_{j} w_{t} /\left(1-w_{i}\right)\left(1-w_{i}-w_{j}\right)$.

From (31), we see that the term $\oplus_{j=1, i \neq j}^{n} n_{j} h_{j}^{q}$ is the weighted power average satisfaction to all criteria except $i$, with $\sum_{j=1, j \neq i}^{n} n_{j}=1$. The term $\oplus_{t=1, t \neq i \neq j}^{n} m_{t} h_{t}^{r}$ is the weighted power average satisfaction of all criteria except $i$ and $j$, with $\sum_{t=1, t \neq j \neq i}^{n} m_{t}=1$. Then, the new operator represents the interrelationship between any three aggregated arguments, which is similar to the GBM. From (32), we see that the term $\oplus_{j, t=1, i \neq j \neq t}^{n} u_{j, t}\left(h_{j}^{q} \otimes h_{t}^{r}\right)$ is the weighted power average satisfaction to any two criteria except $i$ with $\sum_{j, t=1, j \neq t \neq i}^{n} u_{j, t}=$ 1 , which represents another interrelationship form between any three aggregated arguments.

Moreover, based on the operational law of HFEs, HFRWBM, and GHFRWBM, we further derive the following results.

Theorem 9. Let $p, q, r \geq 0$, and $h_{i}(i=1,2, \ldots, n)$ be a collection of HFEs with the weight vector $w=\left(w_{1}, w_{2}, \ldots, w_{n}\right)$ such that $w_{i} \geq 0, w_{i} \neq 1$, and $\sum_{i=1}^{n} w_{i}=1$; then the aggregation value by using the GHFRWBM is a HFE, and

$$
\begin{aligned}
& \text { GHFRWBM } M_{w}^{p, q, r}\left(h_{1}, h_{2}, \ldots, h_{n}\right) \\
& =\left(\bigoplus_{\substack{i, j, t=1 \\
i \neq j \neq t}}^{n} \kappa_{i j t}\right)^{1 /(p+q+r)} \\
& =\bigcup_{k_{i, j, t} \in \kappa_{i j t, i \neq j \neq t}}\left\{\left(1-\prod_{\substack{i, j, t=1 \\
i \neq j \neq t}}^{n}\left(1-k_{i, j, t}\right)\right)^{1 /(p+q+r)}\right\},
\end{aligned}
$$

where $n_{j}=w_{j} /\left(1-w_{i}\right), m_{t}=w_{t} /\left(1-w_{i}-w_{j}\right)$, and $\kappa_{i j t}=w_{i} n_{j} m_{t}\left(h_{i}^{p} \otimes h_{j}^{q} \otimes h_{t}^{r}\right) . \kappa_{i j t}$ can be considered a "bonding satisfaction" factor, capturing the connection among $h_{i}, h_{j}$, and $h_{t}$.

Proof. By the operational laws (1)-(6) of HFEs and (12), we get

$$
\begin{aligned}
\kappa_{i j t}= & w_{i} n_{j} m_{t}\left(h_{i}^{p} \otimes h_{j}^{q} \otimes h_{t}^{r}\right) \\
= & \bigcup_{\substack{r_{i} \in h_{i} \\
r_{j} \in h_{j} \\
r_{t} \in h_{t}}}\left(1-\left(1-r_{i}^{p} r_{j}^{q} r_{t}^{r}\right)^{w_{i} n_{j} m_{t}}\right),
\end{aligned}
$$

which is also a HFE; then (8) can be written as

$$
\begin{aligned}
& \operatorname{GHFRWBM}_{w}^{p, q, r}\left(h_{1}, h_{2}, \ldots, h_{n}\right) \\
& =\left(\bigoplus_{i, j, t=1, i \neq j \neq t}^{n} \kappa_{i j t}\right)^{1 /(p+q+r)} .
\end{aligned}
$$

Furthermore, from the operational law of HFEs, we have

$$
\begin{aligned}
& \left(\bigoplus_{i, j, t=1, i \neq j \neq t}^{n} \kappa_{i j t}\right)^{1 /(p+q+r)} \\
& \quad=\bigcup_{k_{i, j, t} \in \kappa_{i j t, i \neq j \neq t}}\left\{1-\prod_{i, j, t=1, i \neq j \neq t}^{n}\left(1-k_{i, j, t}\right)\right\} .
\end{aligned}
$$

Therefore,

$$
\begin{aligned}
& \operatorname{GHFRWBM}_{w}^{p, q, r}\left(h_{1}, h_{2}, \ldots, h_{n}\right) \\
& =\left(\bigoplus_{\substack{i, j, t=1 \\
i \neq j \neq t}}^{n} \kappa_{i j t}\right)^{1 /(p+q+r)} \\
& =\bigcup_{k_{i, j, t} \in \kappa_{i j t, i \neq j \neq t}}\left\{\left(1-\prod_{\substack{i, j, t=1 \\
i \neq j \neq t}}^{n}\left(1-k_{i, j, t}\right)\right)^{1 /(p+q+r)}\right\} .
\end{aligned}
$$

Then the proof is completed. 
According to (30), we can derive the reducibility, monotonicity, boundedness, and idempotency of the GHFRWBM operator as follows.

Property 5 (reducibility). Let $p, q, r \geq 0$, and $h_{i}(i=$ $1,2, \ldots, n)$ be a collection of HFEs with the weight vector $w=(1 / n, 1 / n, \ldots, 1 / n))$; then

$$
\begin{aligned}
& \operatorname{GHFRWBM}_{w}^{p, q, r}\left(h_{1}, h_{2}, \ldots, h_{n}\right) \\
& =\operatorname{GHFBM}_{w}^{p, q, r}\left(h_{1}, h_{2}, \ldots, h_{n}\right) .
\end{aligned}
$$

Proof. Since $w_{i}=1 / n(i=1,2, \ldots, n)$, then by Definition 8 , we have

$$
\begin{aligned}
& \operatorname{GHFRWBM}_{w}^{p, q, r}\left(h_{1}, h_{2}, \ldots, h_{n}\right) \\
& =\left(\frac{1}{n(n-1)(n-2)}\right. \\
& \left.\quad \times \bigoplus_{i, j, t=1, i \neq j \neq t}^{n}\left(h_{i}^{p} \otimes h_{j}^{q} \otimes h_{t}^{r}\right)\right)^{1 /(p+q+r)} \\
& =\operatorname{GHFBM}_{w}^{p, q, r}\left(h_{1}, h_{2}, \ldots, h_{n}\right),
\end{aligned}
$$

which complete the proof of the property.

Property 6 (monotonicity). Let $h_{\alpha}=\left\{h_{\alpha 1}, h_{\alpha 2}, \ldots, h_{\alpha n}\right\}$ and $h_{\beta}=\left\{h_{\beta 1}, h_{\beta 2}, \ldots, h_{\beta n}\right\}$ be two collections of HFEs, if, for any $\gamma_{\alpha i} \in h_{\alpha i}$ and $\gamma_{\beta i} \in h_{\beta i}$, we have $\gamma_{\alpha i} \leq \gamma_{\beta i}$ for all $i(i=1,2, \ldots, n)$, and thus

$$
\begin{aligned}
& \operatorname{GHFRWBM}_{w}^{p, q, r}\left(h_{\alpha 1}, h_{\alpha 2}, \ldots, h_{\alpha n}\right) \\
& \quad \leq \operatorname{GHFRWBM}_{w}^{p, q, r}\left(h_{\beta 1}, h_{\beta 2}, \ldots, h_{\beta n}\right) .
\end{aligned}
$$

Proof. Using the terms above and (33), we have

$$
\begin{aligned}
(1- & \left.\prod_{\substack{i, j, t=1 \\
i \neq j \neq t}}^{n}\left(1-k_{\alpha_{i, j, t}}\right)\right)^{1 /(p+q+r)} \\
& \leq\left(1-\prod_{\substack{i, j, t=1 \\
i \neq j \neq t}}^{n}\left(1-k_{\beta_{i, j, t}}\right)\right)^{1 /(p+q+r)} .
\end{aligned}
$$

Then,

$$
\begin{aligned}
& \left(\bigoplus_{i, j, t=1, i \neq j \neq t}^{n} \kappa_{\alpha_{i j t}}\right)^{1 /(p+q+r)} \\
& =\bigcup_{k_{\alpha_{i j t}} \in \kappa_{\alpha_{i j} t, i \neq j \neq t}}\left\{\left(1-\prod_{\substack{i, j, t=1 \\
i \neq j \neq t}}^{n}\left(1-k_{\alpha_{i j t}}\right)\right)^{1 /(p+q+r)}\right\} \\
& \left.\leq\left(\bigoplus_{i, j, t=1, i \neq j \neq t}^{n} \kappa_{\beta_{i j}}\right)^{1 /(p+q+r)}\right\} \\
& =\bigcup_{k_{\beta_{i j t}} \in \kappa_{\beta_{i j}, i \neq j \neq t}}^{n}\left\{\left(1-\prod_{\substack{i, j, t=1 \\
i \neq j \neq t}}^{n}\left(1-k_{\beta_{i j t}}\right)\right)^{1 /(p+q+r)}\right\},
\end{aligned}
$$

which complete the proof of the property.

Property 7 (boundedness). Let $p, q, r \geq 0$, and $h_{i}(i=$ $1,2, \ldots, n)$ be a collection of HFEs with the weight vector $w=\left(w_{1}, w_{2}, \ldots, w_{n}\right)$ such that $w_{i} \geq 0, w_{i} \neq 1$, and $\sum_{i=1}^{n} w_{i}=1$; we have

$$
h^{-} \leq \operatorname{GHFRWBM}_{w}^{p, q, r}\left(h_{1}, h_{2}, \ldots, h_{n}\right) \leq h^{+},
$$

where $h^{-}$and $h^{+}$are lower and upper bounds, respectively [8], with $h^{-}=\min _{i}\left\{\gamma_{i} \mid \gamma_{i} \in h_{i}\right\}$ and $h^{+}=\max _{i}\left\{\gamma_{i} \mid \gamma_{i} \in h_{i}\right\}$.

Proof. Since $h^{-} \leq \gamma_{i} \leq h^{+}$, for all $i$, then

$$
\begin{aligned}
\left(h^{-}\right)^{p+q+r} & =\left(h^{-}\right)^{p} \otimes\left(h^{-}\right)^{q} \otimes\left(h^{-}\right)^{r} \\
& \leq h_{i}^{p} \otimes h_{j}^{q} \otimes h_{t}^{r} \leq\left(h^{+}\right)^{p} \otimes\left(h^{+}\right)^{q} \otimes\left(h^{+}\right)^{r} \\
& =\left(h^{+}\right)^{p+q+r}, \\
h^{-} \leq & \left(\bigoplus_{i, j, t=1, i \neq j \neq t}^{n} \frac{w_{i} w_{j} w_{t}}{\left(1-w_{i}\right)\left(1-w_{i}-w_{j}\right)}\right. \\
& \left.\quad \times\left(h_{i}^{p} \otimes h_{j}^{q} \otimes h_{t}^{r}\right)\right)^{1 /(p+q+r)} \leq h^{+} .
\end{aligned}
$$

Therefore,

$$
h^{-} \leq \operatorname{GHFRWBM}_{w}^{p, q, r}\left(h_{1}, h_{2}, \ldots, h_{n}\right) \leq h^{+},
$$

which complete the proof of the property.

Property 8 (idempotency). Let $p, q, r \geq 0$, and $h_{i}(i=$ $1,2, \ldots, n)$ be a collection of HFEs with the weight vector $w=\left(w_{1}, w_{2}, \ldots, w_{n}\right)$ such that $w_{i} \geq 0, w_{i} \neq 1$, and $\sum_{i=1}^{n} w_{i}=1$. If all $h_{i}$ are equal; that is, $h_{i}=h$ for all $i$, then

$$
\operatorname{GHFRWBM}_{w}^{p, q, r}\left(h_{1}, h_{2}, \ldots, h_{n}\right)=h .
$$


Proof. Since $h_{i}=h(i=1,2, \ldots, n)$, then

$$
\begin{aligned}
& \operatorname{GHFRWBM}_{w}^{p, q, r}\left(h_{1}, h_{2}, \ldots, h_{n}\right) \\
& =\left(\bigoplus_{\substack{i, j, t=1 \\
i \neq j \neq t}}^{n} \frac{w_{i} w_{j} w_{t}}{\left(1-w_{i}\right)\left(1-w_{i}-w_{j}\right)}(h)^{p+q+r}\right)^{1 /(p+q+r)} \\
& =\left(h^{p+q}\right)^{1 /(p+q)}=h,
\end{aligned}
$$

which complete the proof of the property.

Now, if we change the parameters $p, q$, and $r$ of the HFRWBM, then we can get some special cases as follows.

Case 1. If $r=0$, based on (30), we have

$$
\begin{aligned}
& \operatorname{GHFRWBM}_{w}^{p, q, r}\left(h_{1}, h_{2}, \ldots, h_{n}\right) \\
& =\operatorname{HFRWBM}_{w}^{p, q}\left(h_{1}, h_{2}, \ldots, h_{n}\right) .
\end{aligned}
$$

Case 2. If $p=0, q=0$, and $r=0$, based on (30), we get

$$
\operatorname{GHFRWBM}_{w}^{0,0,0}\left(h_{1}, h_{2}, \ldots, h_{n}\right)=\bigoplus_{i=1}^{n} w_{i}=1 .
$$

Case 3. If $p=1, q=0$, and $r=0$, based on (30), we have

$$
\begin{aligned}
\operatorname{HFRWBM}_{w}^{1,0,0}\left(h_{1}, h_{2}, \ldots, h_{n}\right) & =\bigoplus_{i}^{n} w_{i} h_{i} \\
& =\operatorname{HFWA}\left(h_{1}, h_{2}, \ldots, h_{n}\right) .
\end{aligned}
$$

Case 4. If $p=1, q=1$, and $r=1$, then (30) is transformed as

$$
\begin{gathered}
\operatorname{GHFRWBM}_{w}^{1,1,1}\left(h_{1}, h_{2}, \ldots, h_{n}\right) \\
=\left(\bigoplus_{i, j, t=1, i \neq j \neq t}^{n} \frac{w_{i} w_{j} w_{t}}{\left(1-w_{i}\right)\left(1-w_{i}-w_{j}\right)}\right. \\
\left.\times\left(h_{i} \otimes h_{j} \otimes h_{t}\right)\right)^{1 / 3},
\end{gathered}
$$

which we call a generalized hesitant fuzzy reducible triple weighted BM (GHFRTWBM).

Case 5. If $p=2, q=0$, and $r=0$, then (30) is transformed as

$$
\operatorname{GHFRWBM}_{w}^{2,0,0}\left(h_{1}, h_{2}, \ldots, h_{n}\right)=\left(\bigoplus_{i=1}^{n} w_{i} h_{i}^{2}\right)^{1 / 2},
$$

which we call a generalized hesitant fuzzy Euclidean distance weighted BM (GHFREWBM).

Based on the above five cases, we find that (1) Case 1 proves that the GHFRWBM is a generalized form of the
HFRWBM and reduces to the HFRWBM when $r=0$; (2) Case 2 presents orthonormality of the GHFRWBM operator; (3) Case 3 indicates the HFWA operator is a special case of the GHFRWBM; (4) Case 4 proposes the simplest interrelated GHFRWBM; (5) Case 5 shows that the GHFRWBM could be used to compute the Euclidean distance between two HFEs.

\section{An Approach to Hesitant Fuzzy Multicriteria Aggregation}

Based on Definitions 6 and 8 and Theorems 7 and 9, below we develop a three-step approach for multicriteria aggregation under hesitant fuzzy environment.

Step 1. For a hesitant fuzzy multicriteria aggregation problem, let $Y=\left\{y_{1}, y_{2}, \ldots, y_{m}\right\}$ be a set of $m$ alternative, and let $C=\left\{c_{1}, c_{2}, \ldots, c_{n}\right\}$ be a set of $n$ criteria, whose weight vector is $w=\left(w_{1}, w_{2}, \ldots, w_{n}\right)^{T}$, such that $w_{j} \geq 0$ and $\sum_{j=1}^{n} w_{j}=1$, where $w_{j}$ denotes the important degree of the criteria $c_{j}$. The decision makers provide all possible values so that the alternative $y_{i}$ satisfies the criteria $c_{j}$ represented by a HFE $h_{i j}=\cup_{r_{i j} \in h_{i j}}\left\{r_{i j}\right\}$ and all $h_{i j}$ construct a hesitant fuzzy multicriteria aggregation matrix $H_{i j}=\left(h_{i j}\right)_{m \times n}$ with $i=1,2, \ldots, m$ and $j=1,2, \ldots, n$.

Step 2. Utilize the HFRWBM or GHFRWBM (here, we recommend to take square or triple operator, respectively; i.e., $p=q=1$ or $p=q=r=1$ ) to aggregate all the performance values $h_{i j}$ of the $i$ th line and get the overall performance value $h_{i}$ corresponding to the alternative $y_{i}$; that is, $h_{i}=\operatorname{HFRWBM}_{w}^{1,1}\left(h_{i 1}, h_{i 2}, \ldots, h_{i n}\right)$ or $h_{i}=$ $\operatorname{GHFRWBM}_{w}^{1,1,1}\left(h_{i 1}, h_{i 2}, \ldots, h_{i n}\right)$.

Step 3. Compute the scores $s\left(h_{i}\right)$ of $h_{i}$ and rank all the alternatives $y_{i}(i=1,2, \ldots, n)$ according to $s\left(h_{i}\right)$ in descending order and choose the optimal alternative.

In the following, we apply the HFRWBM and GHFRWBM to a multicriteria aggregation problem under the hesitant fuzzy environment.

Example. Let us consider a company which intends to rent a house for its local office. Three alternatives $y_{i}(i=1,2,3)$ are available, and the decision makers consider three criteria to decide which house to choose: $c_{1}$ (price), $c_{2}$ (location), and $c_{3}$ (transportation). The weight vector of three criteria is $w=(0.4,0.3,0.3)^{T}[21]$. Assume that the characteristics of the alternatives $y_{i}$ with respect to the criteria $c_{j}(j=1,2,3)$ are represented by HFEs $h_{i j}=\cup_{r_{i j} \in h_{i j}}\left\{r_{i j}\right\}$, where $r_{i j}$ indicates the degree to which the alternative $y_{i}$ satisfies the criterion $c_{j}$. All $h_{i j}$ are contained in a hesitant fuzzy multicriteria aggregation matrix $H_{i j}=\left(h_{i j}\right)_{3 \times 3}$ (see Table 1 ).

In Table 1 , all criteria $c_{j}$ are transformed to the benefit type criteria and do not need normalization. We first utilize the HFRWBM (here, we take $p=q=1$, that is, the HFRSWBM) to aggregate all the performance values $h_{i j}$ of the $i$ th line and get the overperformance value $h_{i}$ corresponding 
TABLE 1: The hesitant fuzzy multicriteria aggregation matrix.

\begin{tabular}{lccc}
\hline Alternatives & \multicolumn{3}{c}{ Criteria } \\
& $c_{1}$ & $c_{2}$ & $\mathcal{c}_{3}$ \\
\hline$y_{1}$ & $(0.68,0.77)$ & $(0.45,0.55,0.60)$ & $(0.82,0.95)$ \\
$y_{2}$ & $(0.72,0.83,0.91)$ & $(0.78)$ & $(0.15,0.27,0.35)$ \\
$y_{3}$ & $(0.21,0.33)$ & $(0.82)$ & $(0.67,0.75)$ \\
\hline
\end{tabular}

to the alternative $y_{i}$; then we calculate the scores of all the alternatives next:

$$
s\left(h_{1}\right)=0.5051, \quad s\left(h_{2}\right)=0.3892, \quad s\left(h_{3}\right)=0.4453 .
$$

Therefore, $y_{1}>y_{3}>y_{2}$, and the alternative $y_{1}$ is the optimal alternative.

Now, we use the GHFRWBM operator (here, we take $p=$ $q=r=1$, i.e., the GHFRSWBM) to aggregate the hesitant fuzzy data in Table 1 and obtain the scores of three alternatives as follows:

$$
s\left(h_{1}\right)=0.2078, \quad s\left(h_{2}\right)=0.1754, \quad s\left(h_{3}\right)=0.1321 .
$$

Therefore, we give the ranking of $y_{i}$ as $y_{1}>y_{2}>y_{3}$, and the alternative $y_{1}$ is still the optimal alternative.

Based on the aforementioned numeral results, there are three phenomena that could be found.

In the first, the whole ranking of the alternatives has changed, despite the fact that the optimal decision has been unchanged. That is because the HFRWBM operator pays more attention to some arguments, whose performances are too high or too low, while the GHFRWBM operator focuses on the whole arguments instead. In other words, the HFRWBM operator reflects the interrelationship between the individual criterion and other criteria, but the GHFRWBM operator presents the interrelationship of any three criteria.

In the second, the aggregated scores by the GHFRWBM are smaller than the corresponding aggregated scores by the HFRWBM. That is because the GHFRWBM operator is a three-layer multiplication calculation and the HFRWBM operator is a two-layer multiplication calculation, which could be seen from (8) and (30). Therefore, we will get the smaller aggregated scores and the lower identification when the GHFRWBM operator is used to aggregate any four or more criteria at once. Just for this, we propose the GHFRWBM operator to use a three-layer multiplication calculation, that is, GHFRWBM ${ }^{p, q, r}$.

In the last, the aggregated hesitant fuzzy values derived by the HFRWBM or GHFRWBM operators depend on the choice of the parameters $p, q$, and $r$. In general, the bigger parameters are, the more calculation effort needed. Then, we recommend taking $p=q=r=1$ and corresponding to the HFRSWBM and GHFRTWBM, respectively, which is not only an intuitive and simple method but also represents an interrelationship of three individual arguments.

\section{Concluding Remarks}

The Bonferroni mean (BM) operator is a traditional mean type aggregation operator bounded by the max and min operators, which can capture the expressed interrelationship of the individual arguments. In this paper, we have extended the weighted BM to accommodate hesitant fuzzy environment and proposed the hesitant fuzzy reducible weighted Bonferroni mean (HFRWBM) and defined the generalized form of this new operator. Next, we have proved and discussed the desirable characteristics of the HFRWBM and GHFRWBM operators which include reducibility, monotonicity, boundedness, and idempotency. Some special cases have been given to represent these desirable characteristics and compare the different HFRWBMs and GHFRWBMs with changed parameters $p, q$, and $r$. It is worth noting that the aggregated hesitant fuzzy values derived by the HFRWBM or GHFRWBM operator depend on the choice of the above parameters, and these parameters are not robust.

In the end, to deal with the situation that the criteria have connections in hesitant fuzzy multicriteria aggregation, a three-step aggregation approach has been proposed on the basis of the HFRWBM and GHFRWBM hesitant fuzzy aggregation operators. Then, an example has been provided to illustrate our results.

\section{Conflict of Interests}

The author declares that there is no conflict of interests regarding the publication of this paper.

\section{Acknowledgments}

This work was supported by National Natural Science Foundation of China (no. 71301141), Humanity and Social Science Youth foundation of Ministry of Education of China (no. 13YJC630247), Science Application Youth Project of Science and Technology Department of Yunnan Province (no. 2013FD029), and Scientific Research Project of talent introduction of Yunnan University of Finance and Economics (no. YC2013D29).

\section{References}

[1] L. A. Zadeh, "The concept of a linguistic variable and its application to approximate reasoning. I," Information Sciences, vol. 8, pp. 199-249, 1975.

[2] D. Dubois and H. Prade, Fuzzy Sets and Systems, vol. 144 of Mathematics in Science and Engineering, Academic Press, New York, NY, USA, 1980.

[3] S. Miyamoto, "Remarks on basics of fuzzy sets and fuzzy multisets," Fuzzy Sets and Systems, vol. 156, no. 3, pp. 427-431, 2005.

[4] R. R. Yager, "On the theory of bags," International Journal of General Systems, vol. 13, no. 1, pp. 23-37, 1987.

[5] K. T. Atanassov, "Intuitionistic fuzzy sets," Fuzzy Sets and Systems, vol. 20, no. 1, pp. 87-96, 1986.

[6] K. Atanassov and G. Gargov, "Interval valued intuitionistic fuzzy sets," Fuzzy Sets and Systems, vol. 31, no. 3, pp. 343-349, 1989. 
[7] V. Torra and Y. Narukawa, "On hesitant fuzzy sets and decision," in Proceedings of the IEEE International Conference on Fuzzy Systems, pp. 1378-1382, Jeju Island, Korea, August 2009.

[8] V. Torra, "Hesitant fuzzy sets," International Journal of Intelligent Systems, vol. 25, no. 6, pp. 529-539, 2010.

[9] Z. Xu and M. Xia, "Distance and similarity measures for hesitant fuzzy sets," Information Sciences, vol. 181, no. 11, pp. 2128-2138, 2011.

[10] Z. Xu and M. Xia, "On distance and correlation measures of hesitant fuzzy information," International Journal of Intelligent Systems, vol. 26, no. 5, pp. 410-425, 2011.

[11] X. Gu, Y. Wang, and B. Yang, "A method for hesitant fuzzy multiple attribute decision making and its application to risk investment," Journal of Convergence Information Technology, vol. 6, no. 6, pp. 282-287, 2011.

[12] W. Zhou and J. M. He, "Intuitionistic fuzzy geometric Bonferroni Mean and their application in multicriteria decision making," International Journal of Intelligent Systems, vol. 27, pp. 995-1019, 2012.

[13] M. Xia and Z. Xu, "Hesitant fuzzy information aggregation in decision making," International Journal of Approximate Reasoning, vol. 52, no. 3, pp. 395-407, 2011.

[14] C. Bonferroni, "Sulle medie multiple di potenze," Bollettino dell'Unione Matematica Italiana, vol. 5, pp. 267-270, 1950.

[15] R. R. Yager, "On generalized Bonferroni mean operators for multi-criteria aggregation," International Journal of Approximate Reasoning, vol. 50, no. 8, pp. 1279-1286, 2009.

[16] W. Zhou and J. M. He, "Intuitionistic fuzzy normalized weighted Bonferroni mean and its application," Journal of Applied Mathematics, vol. 2012, pp. 1-22, 2012.

[17] Z. Xu and R. R. Yager, "Intuitionistic fuzzy bonferroni means," IEEE Transactions on Systems, Man, and Cybernetics B, vol. 41, no. 2, pp. 568-578, 2011.

[18] W. Zhou and J. M. He, "Interval-valued intuitionistic fuzzy ordered precise weighted aggregation operator and its application in group decision making," Technological and Economic Development of Economy, 2014.

[19] M. Xia, Z. Xu, and B. Zhu, "Generalized intuitionistic fuzzy Bonferroni means," International Journal of Intelligent Systems, vol. 27, no. 1, pp. 23-47, 2012.

[20] B. Zhu, Z. Xu, and M. Xia, "Hesitant fuzzy geometric Bonferroni means," Information Sciences, vol. 205, pp. 72-85, 2012.

[21] W. Zhou, "An accurate method for determining hesitant fuzzy aggregation ope rator weights and its application to project investment," International Journal of Intelligent Systems, 2014. 


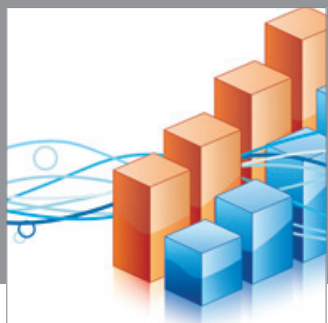

Advances in

Operations Research

mansans

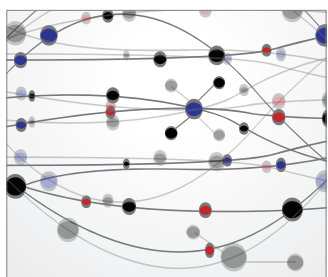

The Scientific World Journal
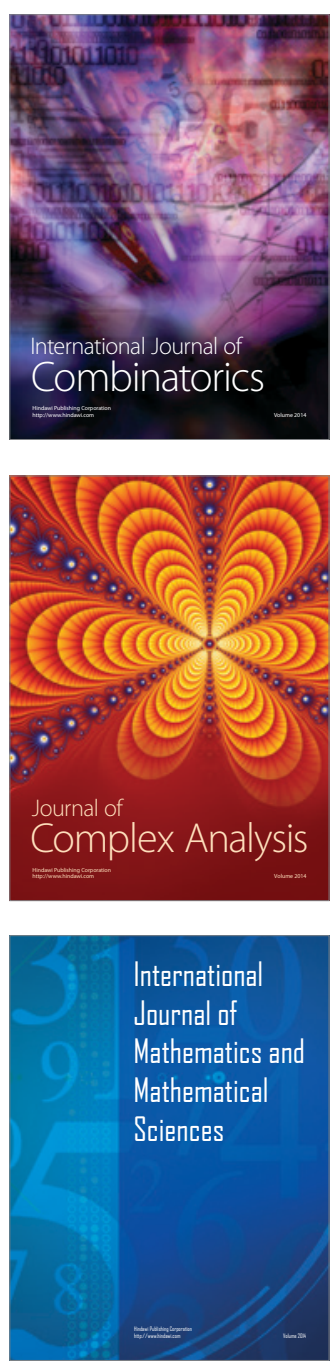
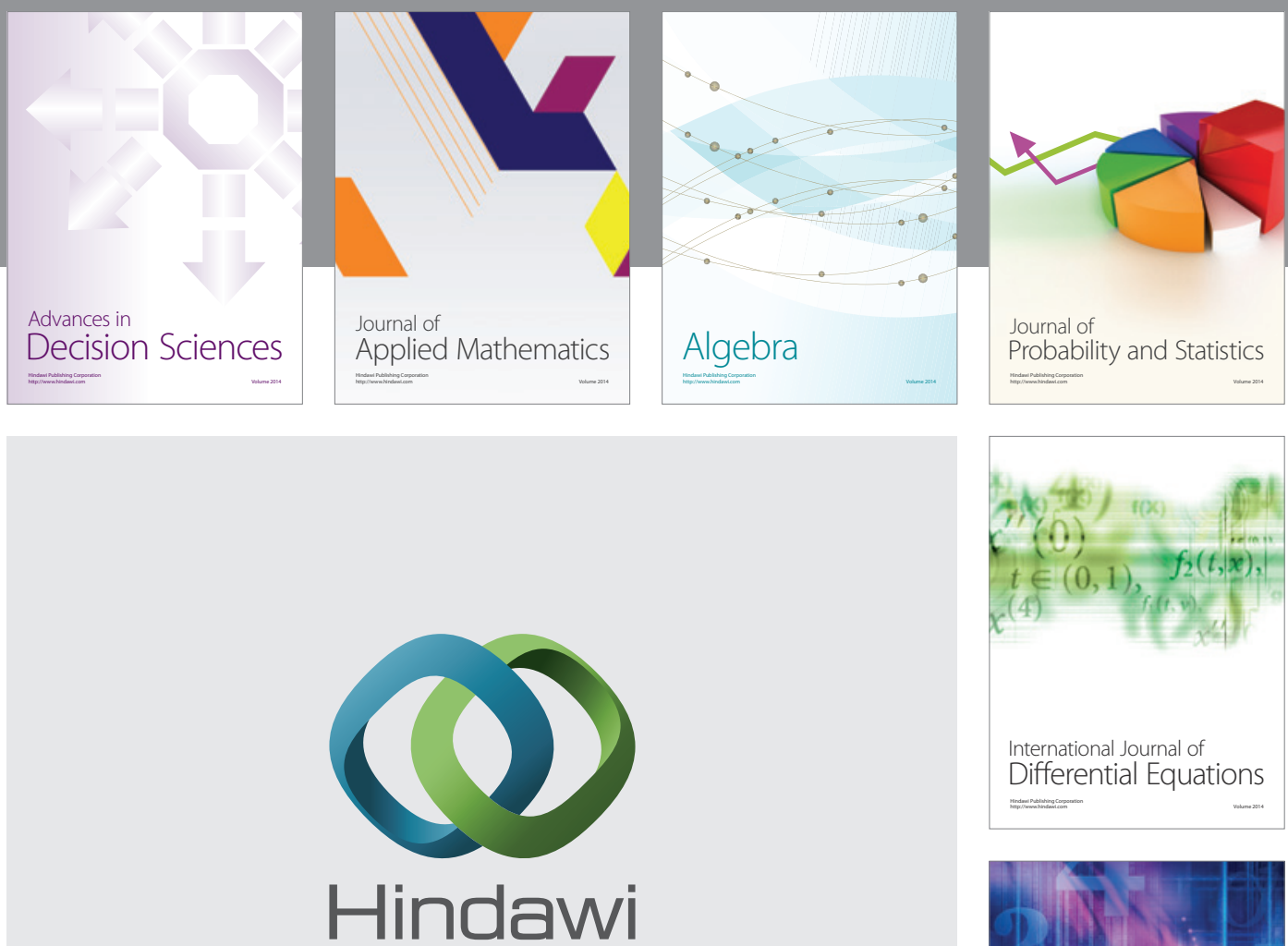

Submit your manuscripts at http://www.hindawi.com
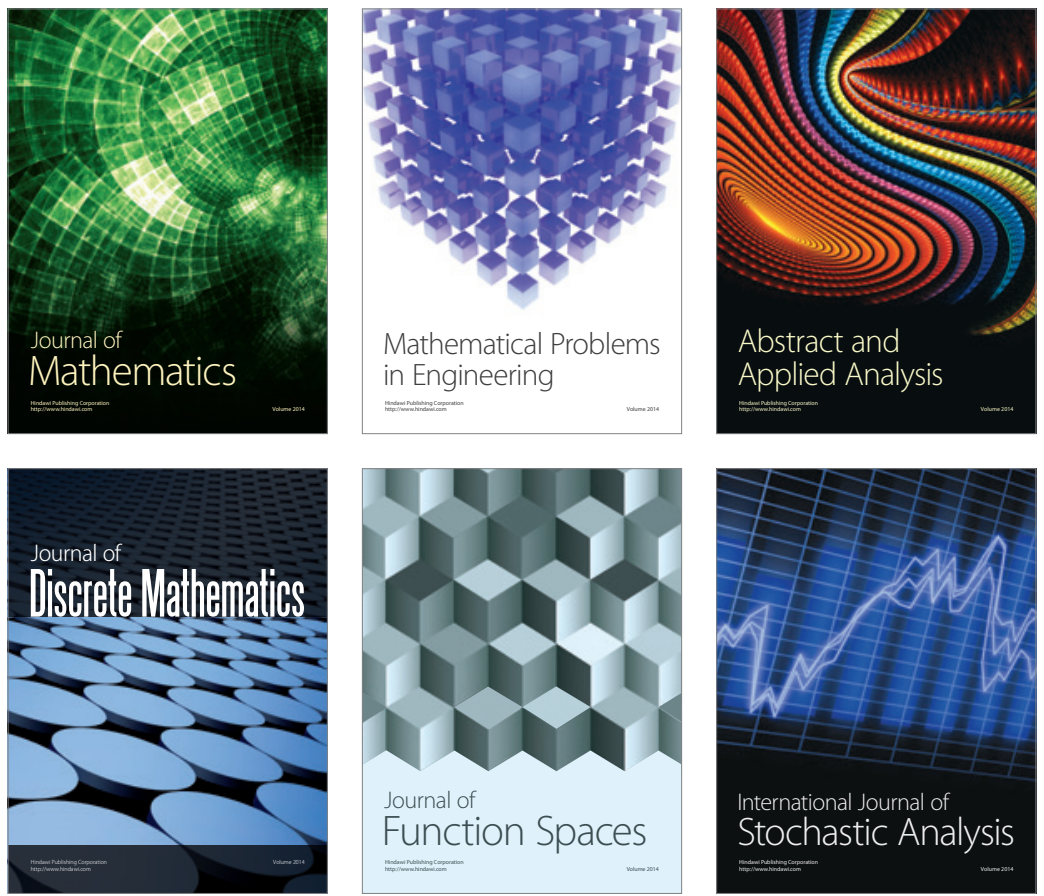

Journal of

Function Spaces

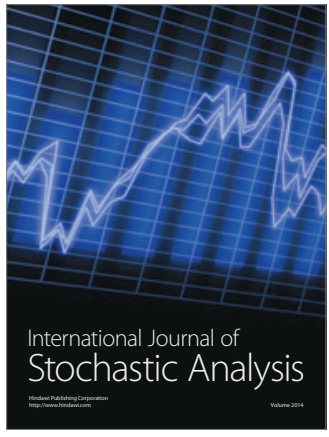

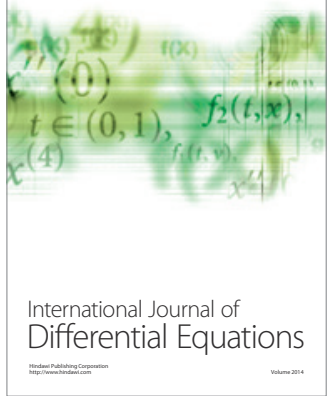
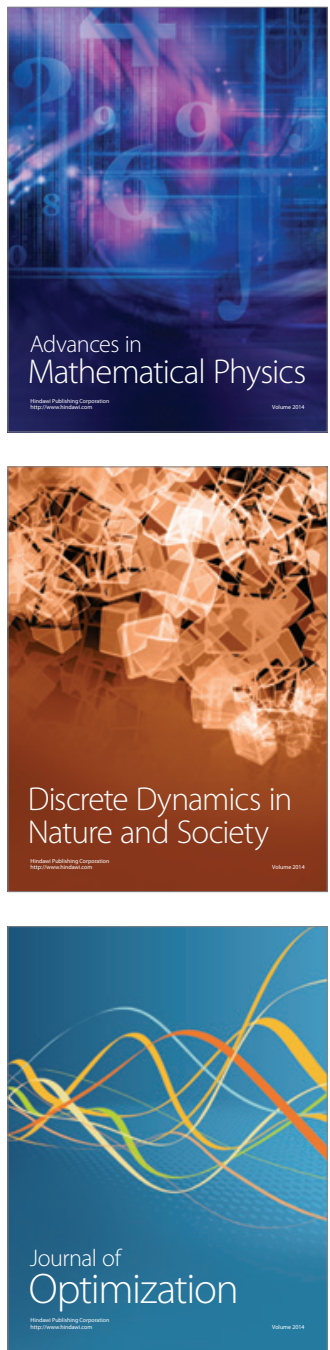